WAVE RADIATION AND WAVE DIFFRACTION FROM A SUBMERGED BODY

IN A UNIFORM CURRENT

by

John Grue and Enok Palm

Department of Mechanics, University of Oslo,

Blindern, Oslo 3, Norway

\title{
ABSTRACT
}

Radiation and diffraction of free surface waves due to a submerged body in aniform current is considered. The fluid layer is infinite deep and the motion is two-dimensional. Applying the method of integral equations, the radiation problem and the diffraction problem for a submerged circular cylindef are examined. For small speed of the current, U, a forced motion of a given frequency will give rise to four waves. It is shown, however, that for circular cylinder, an incoming harmonic wave gives rise to two waves only. Depending on the frequency, the new generated wave may be considered as a transmitted or a reflected wave. The mean second order force is computed. For the radiation problem also the first order damping force is obtained. It is shown that for some values of the parameters, the damping force is negative. This result is closely related to the fact that a harmonic wave traveling upstream with a phase velocity less than $U$, conveys negative energy downstream. 
Reflection of surface waves from a submerged body, based on linearized theory, has been discussed in several papers. One of the most striking results is that in a two-dimensional model of an infinite fluid layer there is no reflection from bodies of circular contour (Dean (1948), Ursell (1950), Grue \& Palm (1984)). The corresponding radiation problem, i.e. the study of the motion generated by a circular cylinder forced to oscillate, has been discussed by Ogilvie (1963). One of his results is that a circular cylinder describing a circular orbit in the clockwise direction, generates at large distances a harmonic wave to the right of the cylinder and no motion to the left. Correspondingly, if the circular orbit is described in the opposite rotational direction, a harmonic wave is generated to the left of the cylinder and no motion to the right. Reversing the wave motion, this means that a circular cylinder describing a circular orbit absorbs an incoming wave completely.

In the present paper we shall generalize the problem by assuming that the water is moving with a uniform velocity $U$. In the frame of reference where the current is zero, we shall thus be studying wave reflection and the radiation problem in connection with a moving submerged body. Only the two-dimensional case will be studied, and in the applications it will be assumed that the submerged body is of a circular form.

The occurrence of a current complicates the problem in various respects. The steady current, as well as the oscillating wave motion, may generate vortex shedding. These effects are disregarded here where we shall focus our interest on the generation of waves. The current also gives rise to steady lee waves (ship waves). These shall shortly be discussed in connection with the restriction they put on the validity of the time-periodic solution. 
A typical feature of waves on a uniform current is that an incoming harmonic wave is split in several waves of different wave lengths, Actually, for weak currents, i.e. for $\tau=U \sigma / g<1 / 4$ ( $\sigma$ the frequency and $g$ the acceleration of gravity), in general four waves will occur. For strong currents, i.e. for $\tau>1 / 4$, two waves occur. A short discussion of the waves will be given in section 4 . The radiation problem will be studied in section 4 and the diffraction problem in section 5 . The solution will be obtained by integral equations. These will be solved partly by an approximate method, valid for deeply submerged bodies, and partly by a numerical method where no such assumptions are made. The approximate method gives the solution of the integral equation immediately. It is shown that the approximate method leads to a fair approximation for circular cylinders with centers placed at depths larger than about twice the radius.

In the radiation problem we shall study sway, heave and the case when the center of the cylinder desribes a circular orbit. The first order damping force and the second order steady force will be computed. It is found that the damping force may be negative for some values of the parameters. This result is shown to be due to the fact that one of the generated waves, the $k_{3}$-wave, conveys negative energy.

In the diffraction problem it will be shown that an incoming wave lgenerates only one new wave for submerged circular bodies, also for $\tau$ < 1/4. An incoming $k_{1}$-wave $\left(k_{2}\right.$-wave) generates a reflecthon wave with wave number $k_{2}\left(k_{1}\right)$. An incoming $k_{3}$-wave or $k_{4}$-wave will be split into a $k_{3}$-wave and a $k_{4}$-wave, with no reflection wave. 


\section{The boundary-value problem}

Let coordinates be taken with the origin 0 in the mean free surface of the fluid, with the $x$-axis horizontal and normal to the generators of the cylinder, and with the y-axis positive upwards. The fluid is assumed incompressible and the motion irrotational. The uniform velocity of the water, $U$, is horizontal and along the negative $x$-axis. The velocity $\vec{v}$ may then be written

$$
\vec{v}=\nabla \Phi^{\star}-\vec{U} \vec{i}
$$

where $\vec{i}$ is the unit vector along the $x$-axis and $\Phi^{\star}$ a velocity potential. $\Phi^{\star}$ satisfies the two-dimensional Laplacian

$$
\nabla^{2} \Phi^{\star}=0
$$

The fluid layer will be assumed to be of infinite depth. The boundary condition at $y=-\infty$ is then

$$
\lim _{y \rightarrow-\infty} \nabla \Phi^{\star}=0
$$

Furthermore, at the submerged body we must have

$$
\frac{\partial \Phi^{\star}}{\partial n}-U n_{x}=v_{n}
$$

where $\partial / \partial n$ denotes the normal derivative and $n_{x}$ is the $x$-compom nent of the normal vector $\vec{n}$ of the body, defined positive into the fluid. $v_{n}$ is the normal velocity of the body. In addition the boundary condition at the free surface must be fulfilled, We shall assume that this condition may be linearized. This is a valid approximation if either the body is deeply submerged or the body is slender. Since we here consider bodies of circular form, the first mentioned condition is assumed satisfied. How deeply the body must be submerged for the assumption to be valid, will be discussed later. The free surface condition then takes the form 


$$
\left(\frac{\partial}{\partial t}-U \frac{\partial}{\partial x}\right)^{2} \Phi^{\star}+g \frac{\partial \Phi^{\star}}{\partial y}=0 \quad y=0
$$

where $t$ denotes time. Besides the boundary conditions (2.3)$(2.5$,$) the radiation conditions at x= \pm \infty$ must be fulfilled.

The solution of the problem is divided into one steady solution and one oscillating part being proportional to $\exp (j \sigma t)$ with $j$ denoting the imaginary unit. We therefore write

$$
\Phi^{\star}=-U \chi(x, y)+\Phi(x, y, t)
$$

where

$$
\Phi(x, y, t)=\operatorname{Re}_{j} \phi(x, y) \exp (j \sigma t)
$$

$R_{j}$ denotes the real part with respect to $j$. We have

$$
\begin{aligned}
& \nabla^{2} \chi=0 \\
& \nabla^{2} \phi=0
\end{aligned}
$$

Assume that the cylinder is oscillating with its centre at

$$
x=\operatorname{Re}_{j}\left(\xi_{x} \exp (j \sigma t)\right), \quad y+d=\operatorname{Re}_{j}\left(\xi_{y} \exp (j \sigma t)\right)
$$

The linearized boundary condition for $\phi$ at the mean position of the body surface is then (see for example Newman (1978) eq. 3.28)

$$
\frac{\partial \phi}{\partial \mathrm{n}}=\overrightarrow{\mathrm{n}} \cdot(j \sigma \vec{\xi}+\mathrm{U}(\vec{\xi} \cdot \nabla) \nabla \chi)
$$

where

$$
\vec{\xi}=\xi_{x} \vec{i}+\xi_{y} \vec{j}
$$

$\vec{j}$ is the unit vector along the y-axis. The last term in (2.11) acapunts for the interaction between the steady and the oscillatory flow fields. The linearized boundary conditions at $y=0$ are

$$
\begin{array}{ll}
U^{2} \frac{\partial^{2} \chi}{\partial x^{2}}+g \frac{\partial \chi}{\partial y}=0 & y=0 \\
\left(j \sigma-U \frac{\partial}{\partial x}\right)^{2} \phi+g \frac{\partial \phi}{\partial y}=0 & y=0
\end{array}
$$


The lee wave problem has been discussed by Lamb (1932, p.410), Havelock (1926, 1936) and Tuck (1965). We shall here mainly be interested in the periodic motion.

The mathematical problem will be solved by transforming it to an integral equation. This may be achieved by expressing $\phi$ as a source distribution over the boundary of the submerged body.

\section{The intergal equation}

The velocity potential for a concentrated source of strength unity, the Green function, oscillating with a frequency $\sigma$ and imbedded in a current of speed $U$, has been derived by Haskind (1954). It is appropriate to rewrite Haskind's form of the Green function so it becomes an analytic function of $z=x+i y$ (we use two imaginary units, $i$ in connection with the space variables and $j$ with the time variable). The Green function for a concentrated source at $z=z_{0}$ is then found to be

$$
G\left(z, z_{0}\right)=\frac{1}{2 \pi}\left[\ln \frac{z-z_{0}}{z-\bar{z}_{0}}-(1-i j) F_{1}(z)-(1+i j) F_{2}(z)\right]
$$

where

$$
\begin{aligned}
& F_{1}(z)=\frac{1}{\sqrt{1-4 \tau}}\left[\int_{\infty}^{z} \frac{\exp \left(-i k_{1}(z-u)\right) d u}{u-\bar{z}_{0}}-\int_{\infty}^{z} \frac{\exp \left(-i k_{2}(z-u)\right) d u}{u-\bar{z}_{0}}\right. \\
& F_{2}(z)=\frac{1}{\sqrt{1+4 \tau}}\left[\int_{\infty}^{z} \frac{\exp \left(-i k_{3}(z-u)\right) d u}{u-\bar{z}_{0}}-\int_{-\infty}^{z} \frac{\exp \left(-i k_{4}(z-u)\right) d u}{u-\bar{z}_{0}}\right.
\end{aligned}
$$

and a bar denotes complex conjungate. With

$$
\nu=\frac{\sigma^{2}}{g}, \tau=\frac{U \sigma}{g}
$$

the four wave numbers are defined by

$$
k_{1,2}=\frac{\nu}{2 \tau^{2}}(1-2 \tau \pm \sqrt{1-4 \tau}), \quad k_{3,4}=\frac{\nu}{2 \tau^{2}}(1+2 \tau \pm \sqrt{1+4 \tau})
$$


The velocity potential $\Phi$ for the motion in consideration may be written

$$
\Phi(x, y, t)=\operatorname{Re}_{i} \operatorname{Re}_{j} f(z) \exp (j \sigma t)
$$

where $f(z)$ is an analytic function. We write

$$
f(z)=f_{0}(z)+f_{1}(z)
$$

where in the diffraction problem $f_{0}(z)$ is the (known) complex potential of the incoming waves. In the radiation problem $f_{0}(z)=0$. $f_{1}(z)$ must satisfy the radiation conditions at $x= \pm \infty$ and the boundary conditions at $y=0$ and $y=-\infty \cdot f_{1}(z)$ is written on the form

$$
f_{1}(z)=\int_{S} \gamma(s) G(z, \zeta(s)) d s
$$

where $s$ is the arclength and $z=\zeta(s)$ is the equation for the contour on parameter form. The source strength $\gamma$ is real with respect to $i . \gamma$ has to be chosen so that the boundary conditions at the body is fulfilled. Let $\beta(s)$ denote the angle between the tangent vector and the $\mathrm{x}$-axis. Hereby

$$
\exp (i \beta(s))=\frac{d \zeta}{d s}
$$

and

$$
f^{\prime}(\zeta(s)) \exp (i \beta(s))=v_{t}(s)+i v_{n}(s)
$$

where $v_{t}$ and $v_{n}$ are the tangential and normal velocity, respectively, Letting $z \rightarrow \zeta$ and applying the boundary condition at the body, the Plemelj formula gives

$$
\begin{array}{r}
\frac{1}{2} i \gamma\left(s^{\prime}\right)+f_{s}(s) G^{\prime}\left(\zeta\left(s^{\prime}\right), \zeta(s)\right) \exp \left(i \beta\left(s^{\prime}\right)\right) d s= \\
v_{t}\left(s^{\prime}\right)+i v_{n}\left(s^{\prime}\right)-f_{0}^{\prime}\left(\zeta\left(s^{\prime}\right)\right) \exp \left(i \beta\left(s^{\prime}\right)\right)
\end{array}
$$


A bar at the integral sign indicates the principal value. Taking the imaginary part of this equation we get rid of $v_{t}$ which is unknown, It is easily shown that this equation is non-singular. This follows from the fact that the only singular term may be written

$$
\operatorname{Im}_{i} \frac{\exp \left(i \beta\left(s^{\prime}\right)\right)}{\zeta\left(s^{\prime}\right)-\zeta(s)}=\frac{\cos \left(n^{\prime}, r\right)}{r}
$$

where $r$ is the radius vector from $\zeta(s)$ to $\zeta\left(s^{\prime}\right)$ and $\left(n^{\prime}, r\right)$ is the angle between the radius vector and the outward normal at the point $\zeta\left(s^{\prime}\right)$, see Grue \& Palm $(1984)$. Since $\cos \left(n^{\prime}, r\right) \rightarrow 0$ as $r \rightarrow 0$ the 1.h.s, of $(3.12)$ is non-singular. The imaginary part of (3.11) becomes

$$
\gamma\left(s^{\prime}\right)+\frac{1}{\pi} \int_{s} \gamma(s) L\left(s^{\prime}, s\right) d s=h\left(s^{\prime}\right)
$$

Here

$$
\begin{aligned}
& L=\operatorname{Im}_{i}\left[\operatorname { e x p } ( i \beta ( s ^ { \prime } ) ) \left(\frac{1}{\zeta\left(s^{\prime}\right)-\zeta(s)}-\frac{1}{\zeta\left(s^{\prime}\right)-\overline{\zeta(s)}}\right.\right.
\end{aligned}
$$

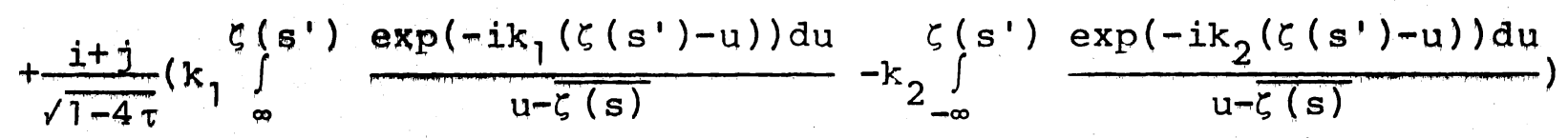

$$
\begin{aligned}
& \left.+\frac{i-j}{\sqrt{1+4 \tau}}\left(k_{3} \int_{\infty}^{\zeta\left(s^{\prime}\right)} \frac{\exp \left(-i k_{3}\left(\zeta\left(s^{\prime}\right)-u\right)\right) d u}{u-\overline{\zeta(s)}}-k_{4} \int_{\infty}^{\zeta\left(s^{\prime}\right)} \frac{\exp \left(-i k_{4}\left(\zeta\left(s^{\prime}\right)-u\right)\right) d u}{u-\zeta(s)}\right)\right)
\end{aligned}
$$

and

$$
h\left(s^{\prime}\right)=2 v_{n}\left(s^{\prime}\right)-2 \operatorname{Im}_{i}\left(f_{0}^{\prime}\left(\zeta\left(s^{\prime}\right)\right) \exp \left(i \beta\left(s^{\prime}\right)\right)\right)
$$

Since there is no net flux of mass transport across the body surface, we have

$$
\int_{s} \gamma(s) d s=\int_{s} v_{n}(s) d s=0
$$

For a body of eircular contour $\cos \left(n^{\prime}, r\right) / r$ in $(3.12)$ is constant and equal to $1 / 2$. From $(3.16)$ it then follows that the contribution from the first term in $(3.14)$ vanishes. 
Let $R$ denote the radius of the cylinder and $d$ the distance between the free surface and the center of the cylinder. When $(d / R)^{2} \gg 1$, the second term in (3.13) may be cancelled (see Grue \& Palm (1984)) and $(3.13)$ reduces to

$$
\gamma\left(s^{\prime}\right)=h\left(s^{\prime}\right)
$$

By using this simplification in the following sections, we are able to obtain convenient, analytical solutions of the problem. We shall, however, for comparison also solve the complete equation (3.13), applying a numerical method. Generally speaking, it will turn out that our approximate solution is a fair approximation when $\mathrm{d} / \mathrm{R}$ is larger than about 2 , provided that $\tau$ is not close to $1 / 4$, To solve the complete equation (3.13) we shall apply Fourier transform. Introducing the angle variable $\theta$ instead of $s$ we have

$$
\zeta(\theta) \equiv i R \exp (i \theta)-i d
$$

where $\theta=0$ corresponds to the uppermost point of the circular contour. We also have

$$
\beta(\theta)=\theta+\pi
$$

According to $(3.16), \gamma(\theta)$ may be written in the form

$$
r(\theta)=\sum_{m=1}^{\infty}\left(c_{m} \exp (i m \theta)+\bar{c}_{m} \exp (-i m \theta)\right)
$$

As shown in the appendix, the Fourier transform of (3.13) is appropriately written as two infinite sets of linear uncoupled equations. It turns out that the systems converge rather rapidly and for almost all purposes in this paper it suffices to truncate the series after ten terms.

We shall especially be interested in the far-field. By contour integration we obtain from (3.1), (3.2), (3.3) and (3.8) that 


$$
\begin{gathered}
\lim _{x \rightarrow \infty} f_{1}(z)=A_{2} \exp \left(-i k_{2} z\right) \\
\lim _{x \rightarrow-\infty} f_{1}(z)=A_{1} \exp \left(-i k_{1} z\right)+A_{3} \exp \left(-i k_{3} z\right)-A_{4} \exp \left(-i k_{4} z\right)
\end{gathered}
$$

where

$$
\begin{array}{lll}
A_{1}=i(1-i j) \frac{1}{\sqrt{1-4 \tau}} \int_{S} \gamma(s) \exp \left(i k_{1} \overline{\zeta(s)}\right) d s & 1=1,2 \\
A_{1}=i(1+i j) \frac{1}{\sqrt{1+4 \tau}} \int_{S} \gamma(s) \exp \left(i k_{1} \overline{\zeta(s)}\right) d s & 1=3,4
\end{array}
$$

Substituting the Fourier series $(3.20)$ in $(3.23)$ and $(3.24) A_{1}$ is obtained in form of infinite series, see appendix (A.14) and (A.15).

\section{The oscillating cylinder}

As a first application we consider the motion generated by an oscillating, submerged circular cylinder. We shall consider four cases: sway, heave and the centre of the cylinder describing a circular orbit clockwise or counter clockwise.

The function $f_{0}(z)$ is now chosen as zero and the r.h.s. of (3.13) reduces to $2 v_{n} \cdot v_{n}$ is equal to $\partial \phi / \partial n$ which is given by (2.11). Furthermore, we have

$$
n_{x}=-\sin \theta, n_{y}=\cos \theta
$$

Hence

$$
2 v_{n}=\xi_{x}\left(-2 j \sigma \sin \theta+2 U \frac{\partial^{2} \chi}{\partial n \partial x}\right)+\xi_{y}\left(2 j \sigma \cos \theta+2 U \frac{\partial^{2} x}{\partial n \partial y}\right)
$$

It is easily shown, utilizing the boundary condition at the body, that

$$
\begin{aligned}
& \mathrm{U} \frac{\partial^{2} x}{\partial \operatorname{n\partial x}}=-\frac{1}{R} \frac{d}{d \theta}\left(v_{t} \sin \theta\right) \\
& U \frac{\partial^{2} x}{\partial n \partial y}=\frac{1}{R} \frac{d}{d \theta}\left(v_{t} \cos \theta\right)
\end{aligned}
$$


where $v_{t}$ is the total tangential velocity in the lee wave problem, given by

$$
v_{t}=-\frac{U}{R} \frac{\partial}{\partial \theta}(x+\chi)
$$

Hence (4.2) only requires the knowledge of $\chi$ along the contour of the body.

As mentioned in the previous section, a fair approximation is obtained by replacing $(3.13)$ by (3.17). With this approximation $x$ is easily found to be given by

$$
\chi=\operatorname{Re}_{i} \frac{R^{2}}{z+i d}
$$

As expected, this is the velocity potential in the infinite field solution, in which the cylinder is replaced by a dipole. From (4,5) it then follows that

$$
v_{t}=2 U \cos \theta
$$

and the right hand side of (3.17) is known. The far-field solution is found from $(3.21)-(3,24)$. The elevation of the free surface, $n$, is found from the Bernoulli equation, assuming that the pressure is zero at the free surface. We have obtained the following results for $\eta_{\infty}$ and $\eta_{-\infty}$ ' the free surface elevation at $x=\infty$ and $x=-\infty$, respectively $(\varepsilon$ is real)

Sway $\left(\xi_{\mathrm{x}}=\varepsilon, \xi_{\mathrm{y}}=0\right):$

$$
\begin{aligned}
& \eta_{\infty}=-a_{2} \cos \left(k_{2} x-\sigma t\right) \\
& \eta_{-\infty}=-a_{1} \cos \left(k_{1} x-\sigma t\right)-a_{3} \cos \left(k_{3} x+\sigma t\right)+a_{4} \cos \left(k_{4} x+\sigma t\right)
\end{aligned}
$$

where

$$
\begin{array}{ll}
a_{1}=\frac{2 \pi\left(k_{1} R\right)^{2} \varepsilon}{\sqrt{T-4 \tau}} \exp \left(-k_{1} d\right), & 1=1,2, \quad \tau<\frac{1}{4} \\
a_{1}=0 \quad 1=1,2, \quad \tau>\frac{1}{4} \\
a_{1}=\frac{2 \pi\left(k_{1} R\right)^{2} \varepsilon}{\sqrt{1+4 \tau}} \exp \left(-k_{1} d\right), & 1=3,4
\end{array}
$$


Heave $\left(\xi_{\mathrm{x}}=0, \xi_{\mathrm{y}}=\varepsilon\right)$ :

$$
\begin{aligned}
& \eta_{\infty}=a_{2} \sin \left(k_{2} x-\sigma t\right) \\
& \eta_{-\infty}=a_{1} \sin \left(k_{1} x-\sigma t\right)+a_{3} \sin \left(k_{3} x+\sigma t\right)-a_{4} \sin \left(k_{4} x+\sigma t\right)
\end{aligned}
$$

The center describes a circular orbit clockwise $\left(\xi_{x}=\varepsilon, \xi_{y}=j \varepsilon\right)$ :

$$
\begin{aligned}
& \eta_{\infty}=-2 a_{2} \cos \left(k_{2} x-\sigma t\right) \\
& \eta_{-\infty}=-2 a_{1} \cos \left(k_{1} x-\sigma t\right)
\end{aligned}
$$

The center describes a circular orbit counter clockwise $\left(\xi_{\mathrm{x}}=\varepsilon\right.$, $\left.\xi_{y}=-j \varepsilon\right):$

$$
\begin{aligned}
& \eta_{\infty}=0 \\
& \eta_{-\infty}=-2 a_{3} \cos \left(k_{3} x+\sigma t\right)+2 a_{4} \cos \left(k_{4} x+\sigma t\right)
\end{aligned}
$$

We see that for $\tau<1 / 4$, four waves with wave numers $k_{1}, k_{2}$ ' $\mathrm{k}_{3}, \mathrm{k}_{4}$ are generated. This is evident from the form of the Green function (3.1). The behaviour of the four waves have been discussed elsewhere in the litterature (see for example Peregrine (1976)). It is appropriate, however, in order to get a better basis for understanding our results, to give a short review of the waves.

For given (positive) $\sigma, k$ is determined by $i . \sigma=U k \pm \sqrt{g k}$ $\left(\mathrm{k}=\mathrm{k}_{4^{\prime}} \mathrm{k}=\mathrm{k}_{3}\right.$ and phase velocity negative) and $i \mathrm{i} \cdot \sigma=\sqrt{\mathrm{gk}}-\mathrm{Uk}$ $\left(\mathrm{k}=\mathrm{k}_{1}, \mathrm{k}=\mathrm{k}_{2}\right.$ and phase velocity positive). The four solutions are indicated in figure 1 .

It is seen from the figure that in order to get four waves, $\sigma$ must be less than a certain maximal value, i.e. $\tau<1 / 4$. For $\tau>1 / 4$ only two waves exist $\left(k_{=}=k_{3}, k_{4}\right)$. The figure also shows that usually $k_{1}, k_{3}$ are much larger than $k_{2}, k_{4}$. Furthermore, the $k_{1}$-wave has a phase velocity greater but a group velocity less than $U$. This wave will therefore travel downstream and finally be one of the waves at $x=-\infty$. As $\tau \rightarrow 1 / 4$, the $k_{1}$-wave and the $k_{2}$-wave merge 
into one wave with a group velocity equal U. This leads to a singularity for $\tau=1 / 4$, revealed in the Green function $(3.1),(3,2)$.

A characteristic feature for the obtained far-field solution for sway and heave, is that the phase difference is exactly $\pi / 2$, just as for $U=0$. This is, however, only an approximate result. It turns out that the solution of the complete equation $(3,13)$ by the procedure sketched in the appendix gives a small additional phase difference. Also the amplitudes are changed by solving the complete equation (3.13). Exact and approximate values of the amplitudes are displayed in figures $2 \mathrm{a}$ and $2 \mathrm{~b}$. We notice, however, that the approximate solution is a good approximation for the considered values of parameters, except for $\tau$ close to $1 / 4$. It is easily shown from (3.13) that $\gamma$ is proportional to $\sqrt{1-4 \tau}$ as $\tau \rightarrow 1 / 4$. It then follows from (3.23) that the amplitude in the exact solution tends towards a finite limit as $\tau \rightarrow 1 / 4$, in opposition to the approximate solution $(4.10 \mathrm{a})$. Thus we have obtained that waves generated by an oscillating body and moving upstream with a group velocity equal to the uniform current, have a finite amplitude. As seen from the figures, the amplitudes become, however, very large close to $\tau=1 / 4$.

The formulas $(4.14)-(4.17)$ may be compared with the corresponding ones obtained by Ogilvie (1963) for $U=0$. It is easily seen that $\tau \neq 0$, the result is rather different for the two directions of revolution. Thus for a clockwise revolution we find for $\tau<1 / 4$ one wave upstream and one wave downstream. For $\tau>1 / 4$ we find no waves at all. For a counter clockwise revolution, however, we obtain no waves at $\mathrm{x}=\infty$ and two waves at $\mathrm{x}=-\infty$.

An estimate of the relative magnitude of the amplitudes $a_{1}$, $a_{2}, a_{3}, a_{4}$ may be found from the approximate formulas (4.10), (4.11), provided that $\tau$ is not close to $1 / 4$. It is seen that the four 
amplitudes depend on $\mathrm{k}$ in the same way, being all proportional to $\mathrm{k}^{2} \exp (-\mathrm{kd})$. Hence all the amplitudes have a maximum value for $k d=2$. Which of the amplitudes is largest, depends on the values of $\sigma$ and $U$ and is determined by (3.5). We also note that the maximum value is larger for $a_{1}, a_{2}$ than for $a_{3}, a_{4}$. We shall then examine the first order damping force and the steady, horizontal second order force for sway and heave. The damping force is found from the far-field solution by applying the energy theorem. The velocity of the body in sway or heave is $-\sigma \varepsilon \sin \sigma t$. The damping force may be written

$$
F=D \sin \sigma t
$$

where $D$ is the transfer function, different for sway and heave. The mean work performed by the damping force on the fluid is then

$$
W=\frac{1}{2} \varepsilon \sigma D
$$

According to the energy equation averaged over a period, we have

$$
W=R_{\infty}-R_{-\infty}
$$

where $R_{x}$ denotes the energy flux. A formula for the energy flux for one harmonic wave on a uniform current has been derived by Longuet-Higgins and stewart (1960). Assuming that the current is along positive $x$-axis, they obtain

$$
R_{X}=\left(U+c_{g}\right) E+S U+\frac{1}{2} \rho h U^{\prime 3}
$$

Here $E$ is the wave energy density, $\rho$ the density of the fluid, $c_{g}$ the group velocity, $s$ the radiation stress, here given by

$$
S=E\left(\frac{2 c g}{c}-\frac{1}{2}\right)
$$

and

$$
U^{\prime}=U+\frac{E}{c \rho h}
$$

where $c$ is the phase velocity and $h$ the depth of the fluid 
layer. E/c $\rho \mathrm{h}$ is the velocity corresponding to the stoke drift. (4.21) has to be modified, partly due to the occurrence of several waves and partly to the fact that there is a mean second order horizontal velocity due to the presence of the body. Due to the last effect we have to add to $(4.21)$ the following term (letting $h \rightarrow \infty)$

$$
\overline{\int_{-\infty}^{\eta}\left(p+\frac{1}{2} \rho \vec{v}^{2}+\rho g y\right) u^{(2)} d y}=\frac{1}{2} \rho U^{2} \int_{-\infty}^{0} \overline{u^{(2)}} d y
$$

where higher order terms at the r.h.s. are neglected. A bar denotes the time average, $\mathrm{p}$ is pressure and $\mathrm{u}^{(2)}$ the second order horizontal velocity. The new term represents the transport of the kinetic energy of the current by the mean second order velocity. The equation of continuity gives



where $\eta^{(1)}$ and $\eta^{(2)}$ are the first and second order surface elevation, respectively, and $u^{(1)}$ the first order horizontal velocity. In $(4.20)$ and $(4.25)$ the effect of the lee waves drop out. Letting now $h \rightarrow \infty$ in (4.21), taking into account that we have several waves, adding (4.24) and applying (4.25) we end up with

$$
\begin{aligned}
D & =\frac{\rho g}{\varepsilon \sigma}\left\{a_{1}^{2}\left(\frac{3}{2} U-c_{g 1}-\frac{U^{2}}{c_{1}}\right)+a_{2}^{2}\left(-\frac{3}{2} U+c_{g 2}+\frac{U^{2}}{c_{2}}\right)\right. \\
& \left.+a_{3}^{2}\left(\frac{3}{2} U-c_{g 3}-\frac{U^{2}}{c_{3}}\right)+a_{4}^{2}\left(\frac{3}{2} U+c_{g 4}+\frac{U^{2}}{c_{4}}\right)\right\}, \quad 0 \leqslant \tau<\frac{1}{4} \\
D & =\frac{\rho g}{\varepsilon \sigma}\left\{a_{3}^{2}\left(\frac{3}{2} U-c_{g 3}-\frac{U^{2}}{c_{3}}\right)+a_{4}^{2}\left(\frac{3}{2} U+c_{g 4}+\frac{U^{2}}{c_{4}}\right)\right\}, \quad \tau>\frac{1}{4}
\end{aligned}
$$

As expected, the expression for $D$ is independent of the $u^{(2)}$. $c_{1}$ and $c_{g l}$ are phase velocities and group velocities given by

$$
c_{1}=\sqrt{g / k_{1}}, \quad c_{g l}=\frac{1}{2} \sqrt{g / k_{1}}, \quad 1=1,2,3,4
$$


Values of the damping force based on (4.26) and (4.27) are displayed in figures $3 a$ and $3 b$ for $d / R=2, U / \sqrt{g R}=0.4$ and $\mathrm{U} / \sqrt{\mathrm{gR}}=1.0$, respectively. We note that the approximate solution gives a fair approximation if $\tau$ is not close to $1 / 4$. In opposition to the approximate solution, the complete solution has no discontinuity for $\tau=1 / 4$. However, for larger values of $d / R$, the damping force may vary rapidly close to $\tau=1 / 4$, as shown in figure $3 \mathrm{c}$.

Figure $3 \mathrm{~b}$ reveals that in a certain $\sigma$-domain, the damping force is negative. This may seem somewhat surprising. Physically it means that in spite of the wave motion being swept downstream over larger and larger areas, energy is conveyed to the oscillating body and not away from it. The explanation is that in this $\sigma$-domain, the $\mathrm{k}_{3}$-wave is the dominating one. Returning to $(4.27)$ we first note that

$$
\frac{3}{2} U-c_{g 3}-\frac{U^{2}}{c_{3}}=\left(U-c_{3}\right)\left(\frac{1}{2}-\frac{U}{c_{3}}\right)<0
$$

Hence the $k_{3}$-wave has a positive energy flux, i.e. towards the body. To balance the energy equation for the wave motion which is spread over a continuously increasing area, the additional energy due to the wave must be negative. This is easily seen to be true. The wave energy is equal to $\frac{1}{2} \rho g a_{3}^{2}$. The coupling energy is negative and equal to

$$
-\rho \overline{u_{-\infty} \eta_{-\infty}}=-\frac{1}{2} \rho \mathrm{U} \sqrt{g k_{3}} a_{3}^{2}
$$

The sum of these contributions is

$$
-\frac{1}{2} \rho \sqrt{\mathrm{gk}} \mathrm{a}_{3}^{2}\left(u-\sqrt{\frac{\mathrm{g}}{\mathrm{k}_{3}}}\right)
$$

which always is negative.

To derive the mean horizontal second order force, $\overline{\mathrm{F}}_{\mathrm{x}}$ ' we apply the momentum equation. We have 


$$
-\overline{\mathrm{F}}_{\mathrm{x}}=\overline{\mathrm{I}}_{\infty}-\overline{\mathrm{I}}_{-\infty}
$$

where $I_{x}$ is the momentum flux defined by

$$
I_{X}=\int_{-\infty}^{\eta}\left(\rho\left(u^{(1)}+u^{(2)}-U\right)^{2}+p\right) d y
$$

It is noted that applying the continuity equation the (unknown). second order terms $\eta^{(2)}$ and $u^{(2)}$ disappear. If, instead of the far field method, we use pressure integration at the body, a term proportional to $\mathrm{Uu}^{(2)}$ appears in the expression for $\overline{\mathrm{F}}_{\mathrm{x}}$, which seems difficult to eliminate. We obtain

$$
\begin{aligned}
\bar{F}_{x}= & -\frac{1}{4} \rho g\left[a_{1}^{2}\left(\frac{U}{c_{g 1}}-1\right)+a_{2}^{2}\left(1-\frac{U}{c_{g 2}}\right)+a_{3}^{2}\left(\frac{U}{c_{g 3}}-1\right)\right. \\
& \left.-a_{4}^{2}\left(1+\frac{U}{c_{g 4}}\right)\right]
\end{aligned}
$$

If the lee waves are taken into account, the term

$$
-\frac{1}{4} p g a_{0}^{2}
$$

has to be added to (4.34). The mean second order force $\bar{F}_{x}$ is displayed in figure 4 for $d / R=2.0$ and $U / \sqrt{g k}=0.4$. The force is small except for $\tau$-values close to $1 / 4$.

\section{The diffraction problem}

As the next application we consider the diffraction of an incoming harmonic wave due to a restrained, submerged cylinder. Let the incoming wave elevation, $\eta_{0}$, be given by

$$
n_{0}=a \sin (k x \pm \sigma t)
$$

The corresponding velocity potential is

$$
\Phi_{0}=a \sqrt{\frac{g}{k}} \delta \exp (k y) \cos (k x \pm \sigma t)=\operatorname{Re}_{i} \operatorname{Re}_{j} f_{0}(z) \exp (j \sigma t)
$$

Here $\delta=1$ for $k=k_{4}$ and $\delta=-1$ for $k=k_{1}, k_{2}, k_{3} \cdot f_{0}(z)$ is given by 


$$
f_{0}(z)=\delta a \sqrt{\frac{g}{k}}(1 \pm i j) \exp (-i k z)
$$

The \pm sign indicates that the phase velocity may be along the negative or positive $x$-axis, respectively.

For a submerged body of arbitrary form there will be a transmitted wave of the same wave number as the incoming wave. In addition, for $\tau<1 / 4$ three new waves are generated and for $\tau>1 / 4$ one new wave is generated. As in the previous section we shall study the mean horizontal second order force. An expression for this may for a submerged body of arbitrarily form be written down immediately from (4.34). Let for example the incoming wave be a $\mathrm{k}_{4}^{-}$ wave. The mean second order force is then obtained from (4.34) by adding a term

$$
-\frac{1}{4} \rho g a^{2}\left(1+\frac{U}{c_{g 4}}\right)
$$

such that

$$
\begin{aligned}
\overline{\mathrm{F}}_{\mathrm{x}}= & -\frac{1}{4} \rho g\left[a_{1}^{2}\left(\frac{U}{c_{g 1}}-1\right)+a_{2}^{2}\left(1-\frac{U}{c_{g 2}}\right)+a_{3}^{2}\left(\frac{U}{c_{g 3}}-1\right)\right. \\
& \left.-\left(a_{4}^{2}-a^{2}\right)\left(1+\frac{U}{c_{g 4}}\right)\right]
\end{aligned}
$$

Here $a_{4}$ is the amplitude of the transmitted wave and $a_{1}, a_{2}, a_{3}$ are the amplitudes of the new generated waves (with wave numbers $\mathrm{k}_{1}, \mathrm{k}_{2}, \mathrm{k}_{3}$, respectively). For $\tau>1 / 4 \mathrm{a}_{1}=\mathrm{a}_{2}=0$. Correspondingly, if the incoming wave is a $k_{1}$-wave, $k_{2}$-wave or $k_{3}$-wave, $\overline{\mathrm{F}}_{\mathrm{x}}$ is obtained from $(4.34)$ by adding

$$
\frac{1}{4} \rho g a^{2}\left(\frac{U}{c_{g 1}}-1\right), \frac{1}{4} \rho g a^{2}\left(1-\frac{U}{C_{g 2}}\right), \frac{1}{4} \rho g a^{2}\left(\frac{U}{c_{g 3}}-1\right)
$$

respectively. The formula for $\overline{\mathrm{F}}_{\mathrm{x}}$ may be simplified by using the energy equation. The energy equation is obtained from (4.26), (4.27) by setting $D=0$ and adding the contribution from the incoming wave. When this is a $\mathrm{k}_{4}$-wave, we must add the term 


$$
-a^{2}\left(\frac{3}{2} U+c_{g 4}+\frac{U^{2}}{c_{4}}\right)
$$

and the energy equation gives

$$
\begin{aligned}
& a_{1}^{2}\left(\frac{3}{2} U-c_{g 1}-\frac{U^{2}}{c_{1}}\right)+a_{2}^{2}\left(-\frac{3}{2} U+c_{g 2}+\frac{U}{c_{2}}\right) \\
& +a_{3}^{2}\left(\frac{3}{2} U-c_{g 3}-\frac{U^{2}}{c_{3}}\right)+\left(a_{4}^{2}-a^{2}\right)\left(\frac{3}{2} U+c_{g 4}+\frac{U}{c_{4}}\right)=0
\end{aligned}
$$

Equation (5.8) may be used to eliminate $a_{4}^{2}-a^{2}$ in (5.5). Similar formulas may be derived from $(4.26),(4.27)$ for incoming $k_{1}$-wave, $k_{2}$-wave and $k_{3}$-wave by adding the energy flux of the proper incoming wave.

Let us now utilize that the submerged body is a circular cylinder. We consider first the case that the incoming wave is a $\mathrm{k}_{3}$-wave or a $\mathrm{k}_{4}$-wave. We then have to use the plus sign in (5.2). The Fourier transform of $f_{0}(z)$ is given in the appendix by (A.12) which according to $(A, 6)$ gives $h_{n}^{(1)}-h_{n}^{(4)}=h_{n}^{(2)}+h_{n}^{(3)}=0$. Hence from (A.2), $C_{n}^{(1)}-C_{n}^{(4)}=C_{n}^{(2)}+C_{n}^{(3)}=0$. Introducing this result in $(A .14)$ we obtain that $A_{1}=A_{2}=0$. We have thus shown that the far-field solution consists of two waves only, viz. a $k_{3}$-wave and a $k_{4}$-wave. Let for example the incoming wave be a $k_{4}$-wave. The solution is then of the form

$$
\begin{aligned}
& \eta_{\infty}=a \sin \left(k_{4} x+\sigma t\right) \\
& \eta_{-\infty}=a_{3} \sin \left(k_{3} x+\sigma t+\delta_{3}\right)+a_{4} \sin \left(k_{4} x+\sigma t-\delta_{4}\right)
\end{aligned}
$$

where $\delta_{3}$ and $\delta_{4}$ are phase constants. The solution for an incoming $k_{3}$-wave is obtained from $(5.9)$ and $(5.10)$ by replacing $k_{4}$ with $k_{3}$ in $(5,9)$. We notice that the incoming wave is split up in two waves, but there is no reflection. The result is valid for all $\tau$-values.

We then assume that the incoming wave is either a $k_{1}$-wave or a $k_{2}$-wave. We must then use the minus sign in (5.2). It is now found 
(from the Appendix) that $h_{n}^{(1)}+h_{n}^{(4)}=h_{n}^{(2)}-h_{n}^{(3)}=0$. From (A.1) it then follows that $C_{n}^{(1)}+C_{n}^{(4)}=C_{n}^{(2)}-C_{n}^{(3)}=0$. Introducing this is (A.15) we conclude that $A_{3}=A_{4}=0$. The far field consists also in this case of two waves only. If the incoming wave is a $k_{1}$-wave, the solution will be of the form

$$
\begin{aligned}
& n_{\infty}=a \sin \left(k_{1} x-\sigma t\right)+a_{2} \sin \left(k_{2} x-\sigma t+\delta_{2}\right) \\
& n_{-\infty}=a_{1} \sin \left(k_{1} x-\sigma t+\delta_{1}\right)
\end{aligned}
$$

For an incoming $\mathrm{k}_{2}$-wave we have

$$
\begin{aligned}
& \eta_{\infty}=a_{2} \sin \left(k_{2} x-\sigma t+\delta_{2}\right) \\
& \eta_{-\infty}=a_{1} \sin \left(k_{1} x-\sigma t+\delta_{1}\right)+a \sin \left(k_{2} x-\sigma t\right)
\end{aligned}
$$

It is here assumed that $\tau<1 / 4$. Hence, for incoming $k_{1}$-waves and $\mathrm{k}_{2}$-waves the motion consists of an incoming wave, a transmitted wave and a reflected wave.

A first estimate of the amplitudes is found by applying the approximation $(3,17)$. The Fourier transform of $\gamma$ is then equal to (A.12). This introduced in (A.14) and (A.15) leads to the following expression for the amplitudes of the new waves

$$
\begin{aligned}
& a_{1}=a \frac{4 \pi k_{1} R}{\sqrt{1-4 \tau}} \exp \left(-\left(k_{1}+k_{2}\right) d\right) I_{1}\left(2 \sqrt{k_{1} k_{2}} R\right) \quad 1=1,2 \\
& a_{1}=a \frac{4 \pi k_{1} R}{\sqrt{1+4 \tau}} \exp \left(-\left(k_{3}+k_{4}\right) d\right) I_{1}\left(2 \sqrt{k_{3} k_{4}} R\right) \quad 1=3,4
\end{aligned}
$$

where $I_{1}$ denotes the modified Bessel function of first kind of order one, Furthermore, the phase constant of the new $k_{1}-, k_{2}-$ and $\mathrm{k}_{3}$-waves are $\pi / 2$ whereas the phase constant of the new $\mathrm{k}_{4}$-wave is $-\pi / 2$. The phase constants of the transmitted waves are

$$
\begin{aligned}
& \delta_{1}=a \frac{4 \pi k_{1} R}{\sqrt{1-4 \tau}} \exp \left(-2 k_{1} d\right) I_{1}\left(2 k_{1} R\right) \quad 1=1,2 \\
& \delta_{1}=a \frac{4 \pi k_{1} R}{\sqrt{1+4 \tau}} \exp \left(-2 k_{1} d\right) I_{1}\left(2 k_{1} R\right) \quad 1=3,4
\end{aligned}
$$


The amplitude of the transmitted wave in this approximation is found to be equal to the amplitude of the incoming wave.

We have also found the amplitudes by solving the complete equation ( 3.13 ) using the method outlined in the appendix. The relative amplitude of the generated $k_{3}$-wave for an incoming $k_{4}$ wave, based on (3.13) and (3.17), are displayed in figure 5a. It is noted that for the considered values of the parameters the approximate solution is a fair approximation. The amplitude of the transmitted wave, $a_{4}$, is found to be very close to $a$. In figure $5 b$ the amplitudes of the reflected and transmitted waves for an incoming $\mathrm{k}_{2}$-wave are displayed. It is noted that $\mathrm{a}_{1} / \mathrm{a}$ is very small and $a_{2} / a$ is very close to unity, except near $\tau=1 / 4$. It follows from the energy equation (5.24) that the exact value of $a_{1}$ is finite for $\tau \rightarrow 1 / 4$, as also indicated by the numerical solution.

The general expression (5.5) for the second order force and the energy equation (5.8) takes a simpler form for a body of circular contour. Considering an incoming $\mathrm{k}_{4}$-wave, the energy equation reduces to

$$
a_{4}^{2}-a^{2}=a_{3}^{2} \frac{k_{4}}{k_{3}}
$$

We note that the amplitude of the transmitted wave is larger than the amplitude of the incoming wave, in spite of the fact that a new wave is generated. Using (5.19), the formula for the second order force reduces to

$$
\tilde{F}_{x}=-\frac{1}{4} \rho g \sqrt{1+4 \tau}\left(1-\frac{k_{4}}{k_{3}}\right) a_{3}^{2}
$$

For an incoming $\mathrm{k}_{3}$-wave, the energy equation is obtained from (5.19) by interchanging the subscripts 3 and 4 . It is found that

$$
\overline{\mathrm{F}}_{\mathrm{X}}=-\frac{1}{4} \rho g \sqrt{1+4 \tau}\left(\frac{\mathrm{k}_{3}}{\mathrm{k}_{4}}-1\right) \mathrm{a}_{4}^{2}
$$


For an incoming $k_{1}$ - wave the energy equation gives

$$
a_{1}^{2}-a^{2}=-a_{2}^{2} \frac{k_{1}}{k_{2}}
$$

The second order force may be written

$$
\bar{F}_{x}=\frac{1}{4} \rho g \sqrt{1-4 \tau}\left(\frac{k_{1}}{k_{2}}-1\right) a_{2}^{2}
$$

It is noted that the force is positive, in spite of an incoming wave travelling downstream. If the incoming wave is a $k_{2}$-wave, the energy equation becomes

$$
a_{2}^{2}-a^{2}=-a_{1}^{2} \frac{k_{2}}{k_{1}}
$$

and

$$
\overline{\mathrm{F}}_{\mathrm{x}}=-\frac{1}{4} \rho g \sqrt{1-4 \tau}\left(1-\frac{\mathrm{k}_{2}}{\mathrm{k}_{1}}\right) \mathrm{a}_{1}^{2}
$$

The force is now negative whereas the incoming wave is travelling along the positive $x$-axis.

It is of interest to compare the second order force $\bar{F}_{\mathbf{x}}$ with the lee wave force $(4,35)$. For the most values of the parameters $\overline{\mathrm{F}}_{\mathrm{X}}$ is relatively small. It is found, however, that the largest force is obtained for an incoming $k_{2}$-wave. This force obtains a maximum value about $0.35 \rho \mathrm{ga}^{2}$ for $R \sigma^{2} / \mathrm{g}=0.08$ and $U / \sqrt{\mathrm{gR}}=0.7$, see figure 6 . We notice that if the amplitude of the incoming wave is about the same as the amplitude of the lee wave, the maximal $\overline{\mathrm{F}}_{\mathrm{X}}$ is of the same magnitude as the lee wave force.

6. Discussion

To examine closer the validity of our solution, we have computed the lee wave field and found the maximum value of $|\partial x / \partial x|$ at the free surface. It turns out that the maximum value is close 
to the maximum value at $x=-\infty$, which is displayed in figure 7 . In order that the solution is a uniformly good approximation, this maximum value should be considerably smaller than 1. We notice from the figure that the maximurn of $|\partial \chi / \partial x|$ is in fact larger than 1 for $d / R=2$ and $U / \sqrt{g R}$ near unity. Our solution is most likely no good approximation for $U / \sqrt{g R}$ near unity and $d / R$ smaller than about 4. The figure also indicates that the linearized lee wave problem may with fair approximation be solved by applying (3.17), instead of the complete integral equation.

We have tacitly assumed that (3.13) has a unique solution, which is confirmed by the numerical results. This is easily shown to be true if we assume that the physical problem is unique, which seems to be obvious. Following an argument given by Ursell (1973) in another context, we assume for the moment that $v_{n}=0$ (the body at rest) and there is no incoming wave. According to our basic assumption there is then no motion, and $f_{1}(z)$ is constant outside the body. The right hand side of (3.13) is zero and we shall prove that $\gamma=0$, Let us consider the potential defined by (3.8) inside the body. It is immediately seen that $f_{1}(z)$ is continuous across the boundary. Hence $f_{1}(z)$ inside the body is constant, i.e, $f_{j}^{\prime}(z)=0$. Approaching the boundary from the inside and applying the Plemelj theorem we then have

$$
\operatorname{Im}_{i}\left(2 f_{j}^{\prime}\left(\zeta\left(s^{\prime}\right)\right) \exp \left(i \beta\left(s^{\prime}\right)\right)\right)=-\gamma\left(s^{\prime}\right)+\frac{1}{\pi} \int_{s} \gamma(s) L\left(s^{\prime}, s\right) d s=0 \quad(6,1)
$$

Comparing with $(3.13)$ with the r.h.s. zero, it follows that $\gamma=0$. Hence we have proved the assertion. 


\section{REFERENCES}

Dean, W.R., on the reflection of surface waves by a circular cylinder. Proc.Camb.Phil.Soc., 1948, 44.

Grue, J. and Palm, E., Reflection of surface waves by submerged cylinders, Applied Ocean Res., 1984. (To appear.)

Haskind, M.D., On wave motion of a heavy fluid. (R) Prikl.Mat.Mekh, 1954,18, p. 15 .

Havelock, T.H., The method of images in some problems of surface waves. Proc.Roy.SOC.A., 1926, 115, p. 268.

Havelock, T.H., The forces on a circular cylinder submerged in a uniform stream. Proc.Roy.Soc.A., 1936, 157, p.526.

Lamb, H,, Hydrodynamics, 6th ed. Cambridge University Press, 1932. Longuet-Higgins, M.S. and Stewart, R.W., Changes in the form of short gravity waves or long waves and tidal currents. J.Fluid Mech, 1960, 8 .

Newman, J.N., The theory of ship motions. Adv.Appl.Mech., 1978, 18, p. 221 .

Ogilvie, T.F., First- and second order forces on a cylinder submerged under a free surface. J.Fluid Mech., 1963, 16.

Peregrine, D.H., Interaction of water waves and currents. Adv.Appl.Mech., 1976, 16, p. 9.

Tuck, E.O., The effect of non-linearity at the free surface " on flow past a submerged cylinder. J.Fluid Mech., 1965, 22.

Ursell, F,. Surface waves on deep water in presence of a submerged circular cylinder. Proc.Camb.Phil.Soc., 1950, 46.

Ursell, F., on the exterior problems of acoustics. Proc.Camb.Phil. Soc., 1973, 74, p. 117 . 
APPENDIX, Solution of the integral equation by Fourier transform.

The exact solution of the integral equation (3.13) is obtained using Fourier transform. The Fourier series (3.20) for the unknown $\gamma$ and the parameter forms (3.18) and (3.19) for the contour and the angle $\beta$ are used. Multiplying the integral equation with $\frac{1}{2 \pi} \exp \left(-\operatorname{in} \theta^{\prime}\right)$ and integrating from 0 to $2 \pi$ the following two infinite sets of equations are obtained

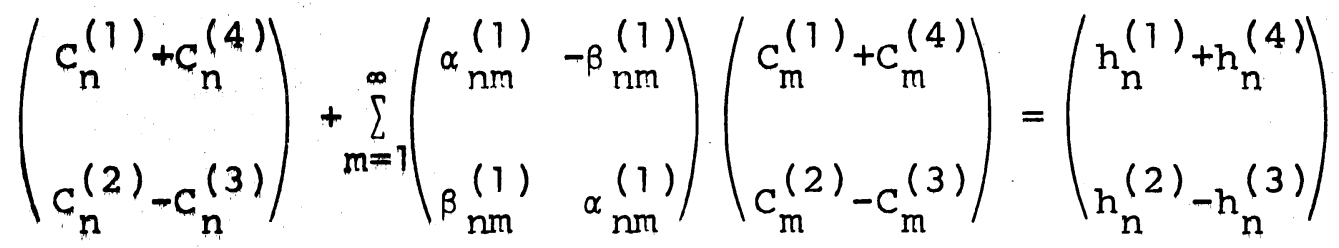

$$
\begin{aligned}
& \left(\begin{array}{l}
C_{n}^{(1)}-C_{n}^{(4)} \\
C_{n}^{(2)}+C_{n}^{(3)}
\end{array}\right)+\sum_{m=1}^{\infty}\left(\begin{array}{cc}
\alpha_{n m}^{(2)} & -\beta_{n m}^{(2)} \\
\beta_{n m}^{(2)} & \alpha_{n m}^{(2)}
\end{array}\right)\left(\begin{array}{l}
C_{m}^{(1)}-C_{m}^{(4)} \\
C_{m}^{(2)}+C_{m}^{(2)}
\end{array}\right)=\left(\begin{array}{l}
h_{n}^{(1)}-h_{n}^{(4)} \\
h_{n}^{(2)}+h_{n}^{(3)}
\end{array}\right)
\end{aligned}
$$

where

$$
\begin{gathered}
{ }_{n}^{(1)}+i \beta{ }_{n m}(1)=\frac{(m+n-1) !}{m !(n-1) !}\left(\frac{R}{2 d}\right)^{m+n}+\frac{2}{\sqrt{1+4 \tau}}\left(\frac{R}{2 d}\right)^{m} \frac{1}{(n-1) !} \times \\
\times\left\{\left(R k_{3}\right)^{n}\left(\exp \left(-2 k_{3} d\right) E_{m+1}\left(2 k_{3} d \exp (-i \pi)\right)+\sum_{q=1}^{n-1} \frac{(m+q-1) !}{m !} \frac{1}{\left(2 k_{3} d\right)^{q}}\right)\right. \\
\left.-\left(R k_{4}\right)^{n}\left(\exp \left(-2 k_{4} d\right) E_{m+1}\left(2 k_{4} d \exp (-i \pi)\right)+\sum_{q=1}^{n-1} \frac{(m+q-1) !}{m !} \frac{1}{\left(2 k_{4} d\right)^{q}}\right)\right\} \quad(A, 3) \\
{ }_{n m}^{(2)}+i \beta{ }_{n m}^{(2)}=\frac{(m+n-1) !}{m !(n-1) !}\left(\frac{R}{2 d}\right)^{m+n}+\frac{2}{\sqrt{1-4 \tau}}\left(\frac{R}{2 d}\right)^{m} \frac{1}{(n-1) !} \times \\
\times\left\{\left(R k_{1}\right)^{n}\left(\exp \left(-2 k_{1} d\right) E_{m+1}\left(2 k_{1} d \exp (-i \pi)\right)+\sum_{q=1}^{n-1} \frac{(m+q-1) !}{m !} \frac{1}{(2 k, d)^{q}}\right)\right. \\
-\left(R k_{2}\right)^{n}\left(\exp \left(-2 k_{2} d\right) E_{m+1}\left(2 k_{2} d \exp (i \pi)\right)+\sum_{q=1}^{n-1} \frac{(m+q-1) !}{m !} \frac{1}{\left.\left.\left(2 k_{2} d\right)^{q}\right)\right\}}\right. \text { (A.4) }
\end{gathered}
$$




$$
\begin{aligned}
& c_{n}=c_{n}^{(1)}+i c_{n}^{(2)}+j c_{n}^{(3)}+i j c_{n}^{(4)} \\
& h_{n}=h_{n}^{(1)}+i h_{n}^{(2)}+j h_{n}^{(3)}+i j h_{n}^{(4)}
\end{aligned}
$$

$h_{n}$ is the Fourier transform of the r.h.s. of (3.13).

$$
h_{n}=\frac{1}{2 \pi} \int_{0}^{2 \pi} h\left(\theta^{\prime}\right) \exp \left(-\operatorname{in} \theta^{\prime}\right) d \theta^{\prime}
$$

and $c_{n}^{(1)}, h_{n}^{(1)}, 1=1,2,3,4$ are reals.

The exponential integral $\mathrm{E}_{\mathrm{m}+1}$ of order $\mathrm{m}+1$ is defined in Abramowitz and stegun (1970) by

$$
E_{m+1}(z)=\frac{(-z)^{m}}{m !}\left(-\ln z-\gamma+\sum_{q=1}^{m} \frac{1}{q}\right)-\sum_{\substack{q=0 \\ q \neq m}}^{\infty} \frac{(-z)^{q}}{q !(q-m)}
$$

where $\gamma=0.5772157$ is Eulers constant.

The systems of equations (A.1) and (A.2) contain the two limits $U \rightarrow 0\left(k_{1}, k_{3} \rightarrow \infty, k_{2} k_{4} \rightarrow v\right)$ and the lee wave problem $\sigma \rightarrow 0$ $\left(k_{1}, k_{3} \rightarrow g / u^{2}, k_{2}, k_{4} \rightarrow 0\right)$.

The Fourier transform of the various right hand sides are given by:

The lee wave problem: $f_{0}(z)=-U z$

$$
h_{n}=i U \delta_{n 1}
$$

where $\delta_{\mathrm{nl}}$ is the Kronecker delta.

The sway problem: $2 v_{n}=\xi_{x}\left(-2 j \sigma \sin \theta+2 U \frac{\partial^{2} \chi}{\partial n \partial x}\right)$

$$
h_{n}=\xi_{x}\left[j j \sigma \delta_{n 1}-\frac{U}{R} n\left(T_{n-1}-T_{n+1}\right)\right] \quad n \geqslant 1
$$

The heave problem: $2 v_{n}=\xi_{y}\left(2 j \sigma \cos \theta+2 U \frac{\partial^{2} \chi}{\partial n \partial y}\right)$

$$
h_{n}=\xi_{y}\left[j \sigma \delta_{n_{1}}+i \frac{U}{R} n\left(T_{n-1}+T_{n+1}\right)\right] \quad n \geqslant 1
$$


The diffraction problem: $f_{0}(z)=\delta \mathrm{a} \sqrt{\frac{g}{k}}(1 \pm i j) \exp (-i k z)$

$$
n_{n}=-(1 \pm i j) \delta a \sqrt{g k} \exp (-k d) \frac{(k R)^{n-1}}{(n-1) !} \quad n>1
$$

In the formulas $(A .10)$ and (A.11) the tangential velocity of the lee wave problem $\left(T_{0}=0\right)$

$$
v_{t}=U \sum_{m=1}^{\infty}\left(T_{m} \exp (i m \theta)+\bar{T}_{m} \exp (-i m \theta)\right)
$$

is used. It may be shown that $T_{n}=-i C_{n}$ where $c_{n}$ is the Fourier transform of $\gamma$ in the lee wave problem.

The amplitudes of the far-field solution for the velocity potential $f_{1}(z)$ are given by the following infinite series

$$
\begin{gathered}
A_{1}=i(1-i j) \frac{2 \pi R \exp \left(-k_{1} d\right)}{\sqrt{1-4 \tau}} \sum_{m=1}^{\infty} \frac{\left(k_{1} R\right)^{m}}{m !}\left(c_{m}^{(1)}-c_{m}^{(4)}\right. \\
\left.+i\left(c_{m}^{(2)}+c_{m}^{(3)}\right)\right) \quad l=1,2 \\
A_{1} i(1+i j) \frac{2 \pi R \exp \left(-k_{1} d\right)}{\sqrt{1+4 \tau}} \sum_{m=1}^{\infty} \frac{\left(k_{1} R\right)^{m}}{m !}\left(c_{m}^{(1)}+c_{m}^{(4)}\right. \\
\left.+i\left(c_{m}^{(2)}-c_{m}^{(3)}\right)\right) \quad l=3,4
\end{gathered}
$$

\section{REFERENCE}

Abramowitz, M and stegun, I.A., Handbook of Mathematical functions. Dover Publications, Inc., New York 1972. 


\section{FIGURE CAPTIONS}

Fig. 1. The four wave numbers for given $\sigma$ and $U$.

Fig. 2a. Amplitudes for radiated waves in the sway problem, $\mathrm{d} / \mathrm{R}=2.0, \mathrm{U} / \sqrt{\mathrm{gR}}=0.4 . \mathrm{a}_{3}$ is approximately zero.

Fig. 2b, Amplitudes for radiated waves in the sway problem, $\mathrm{d} / \mathrm{R}=2.0, \mathrm{U} / \sqrt{\mathrm{gR}}=1.0$.

Fig. 3a. Damping transfer functions in sway and heave, $\mathrm{d} / \mathrm{R}=2.0, \mathrm{U} / \sqrt{\mathrm{gR}}=0.4$.

Fig. 3b. Damping transfer functions in sway and heave, $d / R=2.0, U / \sqrt{g R}=1.0$.

Fig. 3c. Damping transfer functions in sway and heave, $\mathrm{d} / \mathrm{R}=3,0, \mathrm{U} / \sqrt{\mathrm{gR}}=0.4$.

Fig. 4. Mean second order horizontal force in sway and heave, $\mathrm{d} / \mathrm{R}=2.0, \mathrm{U} / \sqrt{\mathrm{gR}}=0.4$.

Fig. 5a. Amplitude of new $k_{3}$-wave in the diffraction problem when $k_{4}$-waves are incident upon the cylinder, $d / R=2.0$, $\mathrm{U} / \sqrt{\mathrm{gR}}=1.0,1.6$.

Fig. 5b. Amplitude of transmitted $k_{2}$-wave and reflected $k_{1}$-wave when $k_{2}$-waves are incident upon the cylinder, $d / R=2.0$, $\mathrm{U} / \sqrt{\mathrm{gR}}=0.4,1.0$.

Fig. 6. Mean second order horizontal force when $\mathrm{k}_{2}$-waves are incident upon the cylinder, $d / R=2.0, U / \sqrt{\mathrm{gR}}=0.7$.

Fig. 7. Maximum of $|\partial x / \partial x|$ at $x=-\infty, d / R=2.0,3.0,4.0$, 


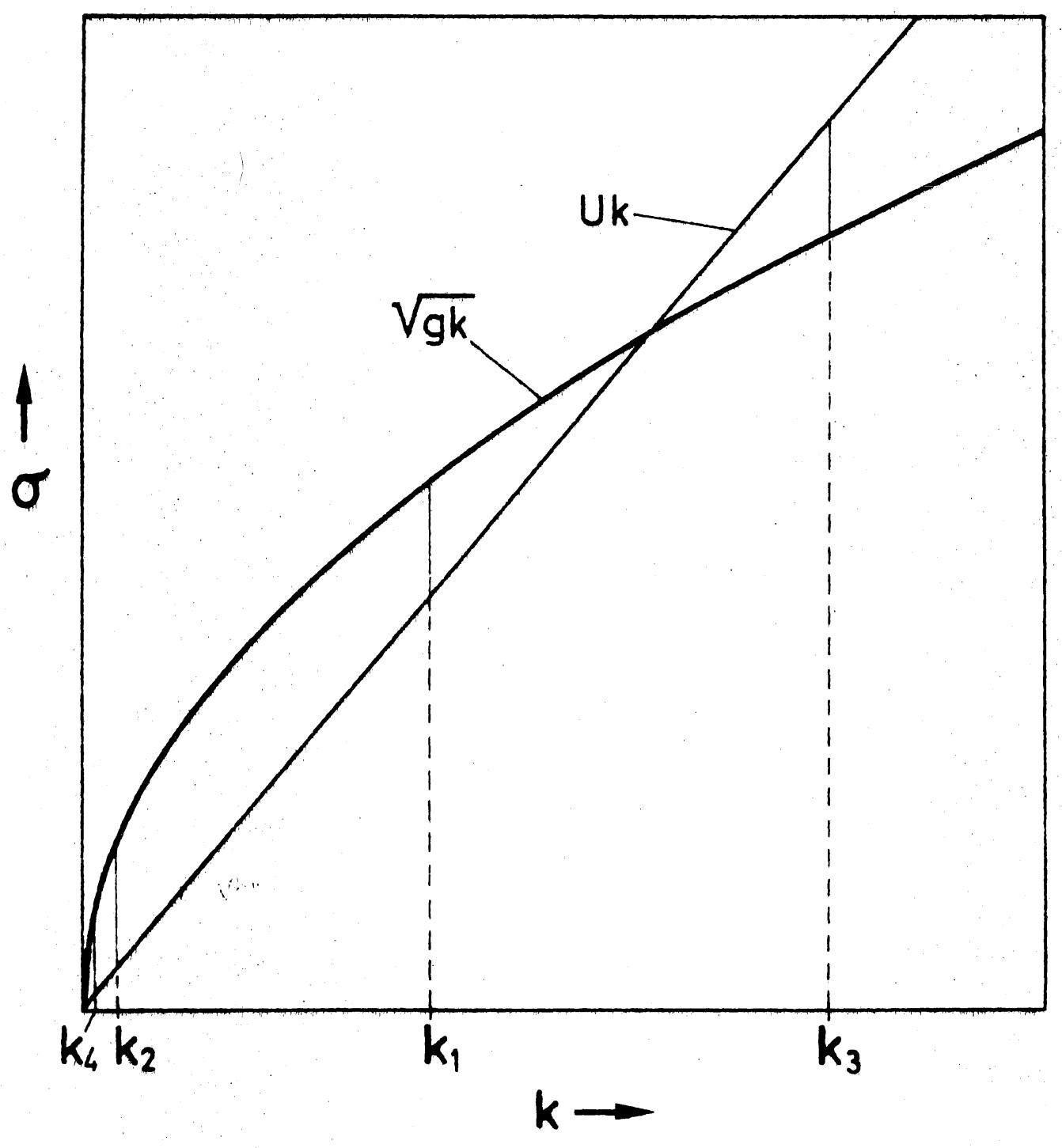

Figure 1. The four wave numbers for given $\sigma$ and $U$, 



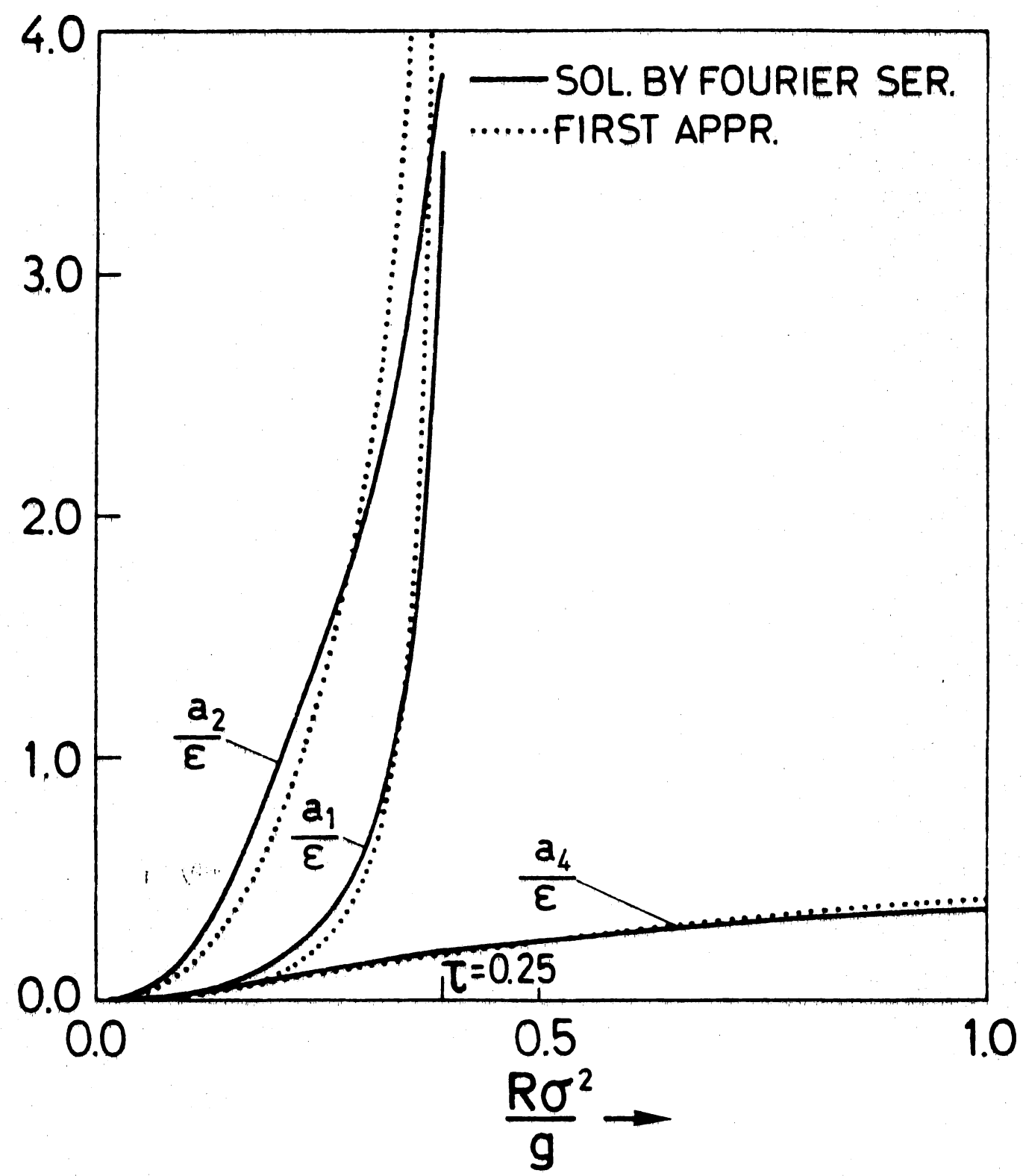

Figure 2a. Amplitudes for radiated waves in the sway problem, $\mathrm{d} / \mathrm{R}=2.0, \mathrm{U} / \sqrt{\mathrm{gR}}=0.4 . \mathrm{a}_{3}$ approximately zero. 



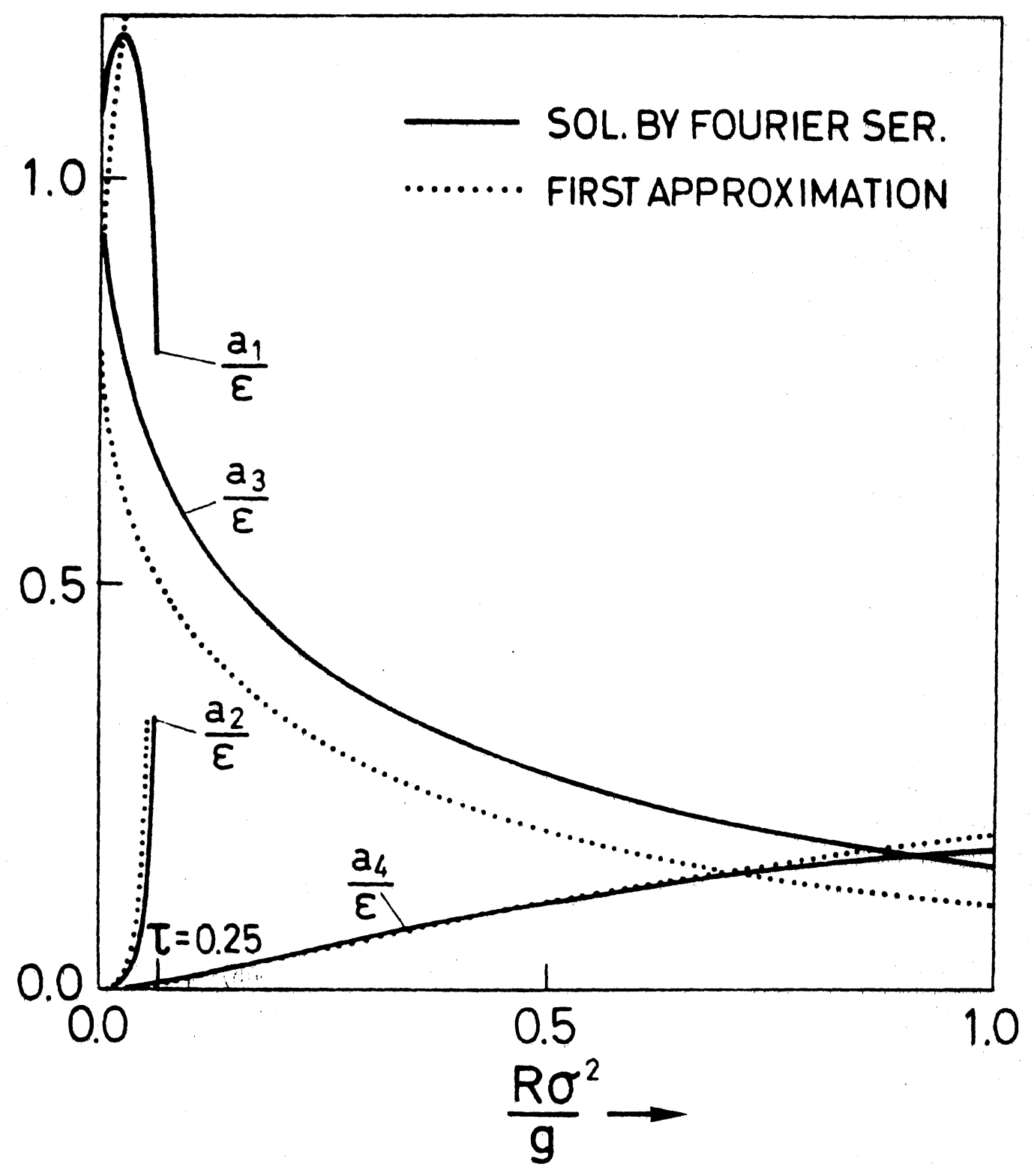

Figure $2 b$. Amplitudes for radiated waves in the sway problem, $\mathrm{d} / \mathrm{R}=2.0, \mathrm{U} / \sqrt{\mathrm{gR}}=1.0$. 



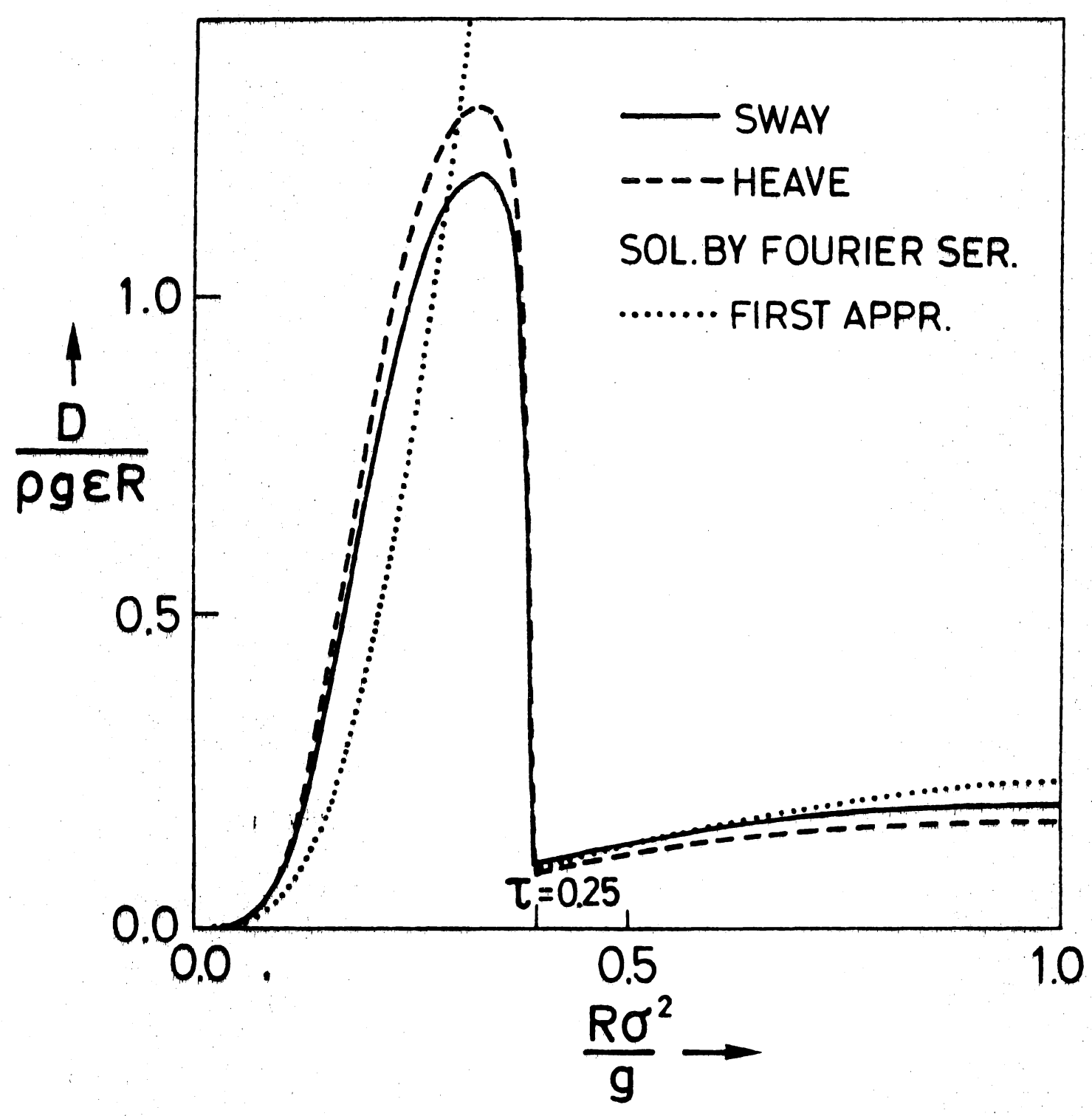

Fiqure 3a. Damping transfer functions in sway and heave, $d / R=2.0, U / \sqrt{g R}=0.4$. 



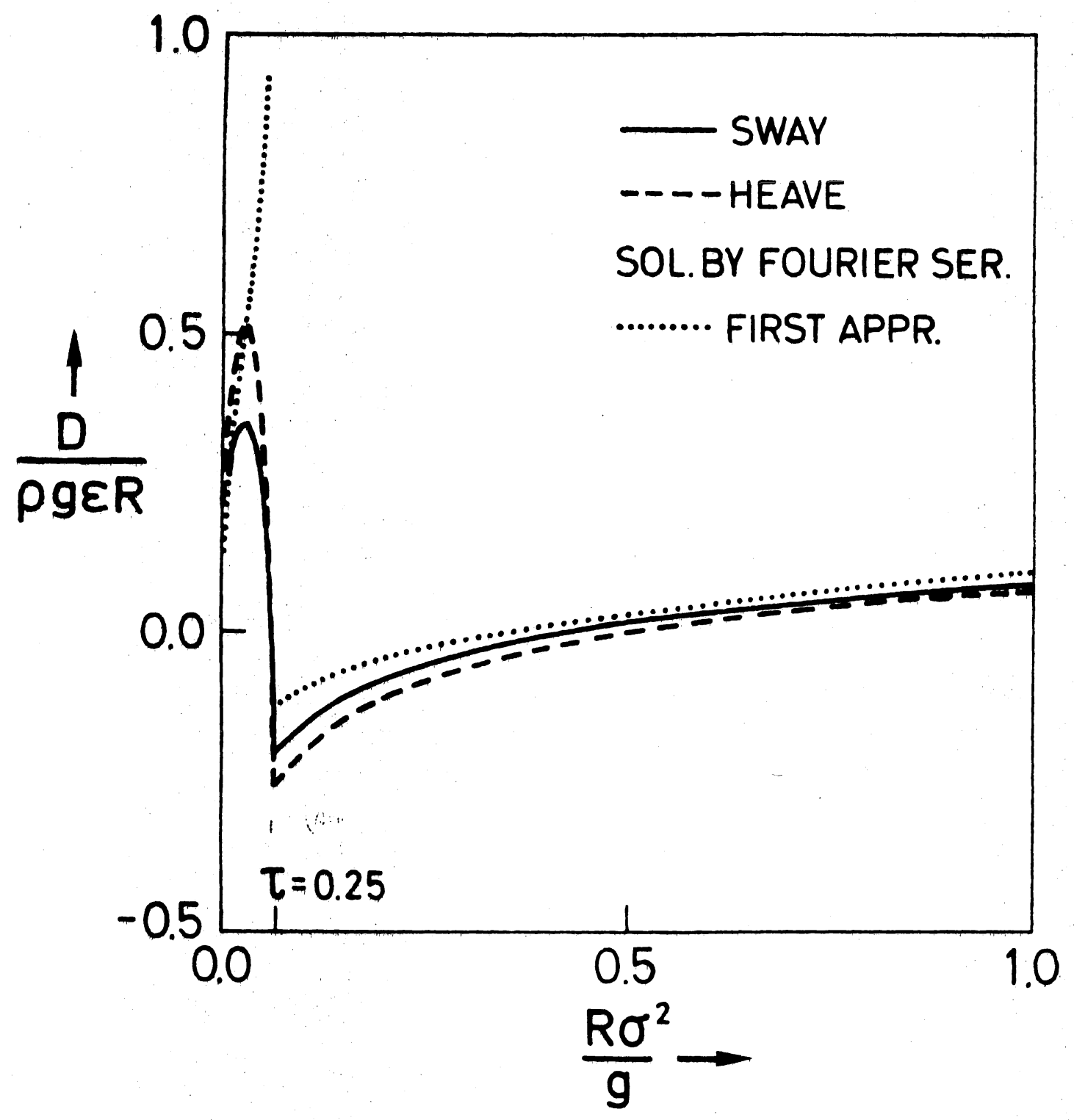

Figure $3 b$. Damping transfer functions in sway and heave, $d / R=2.0, U / \sqrt{g R}=1.0$ 



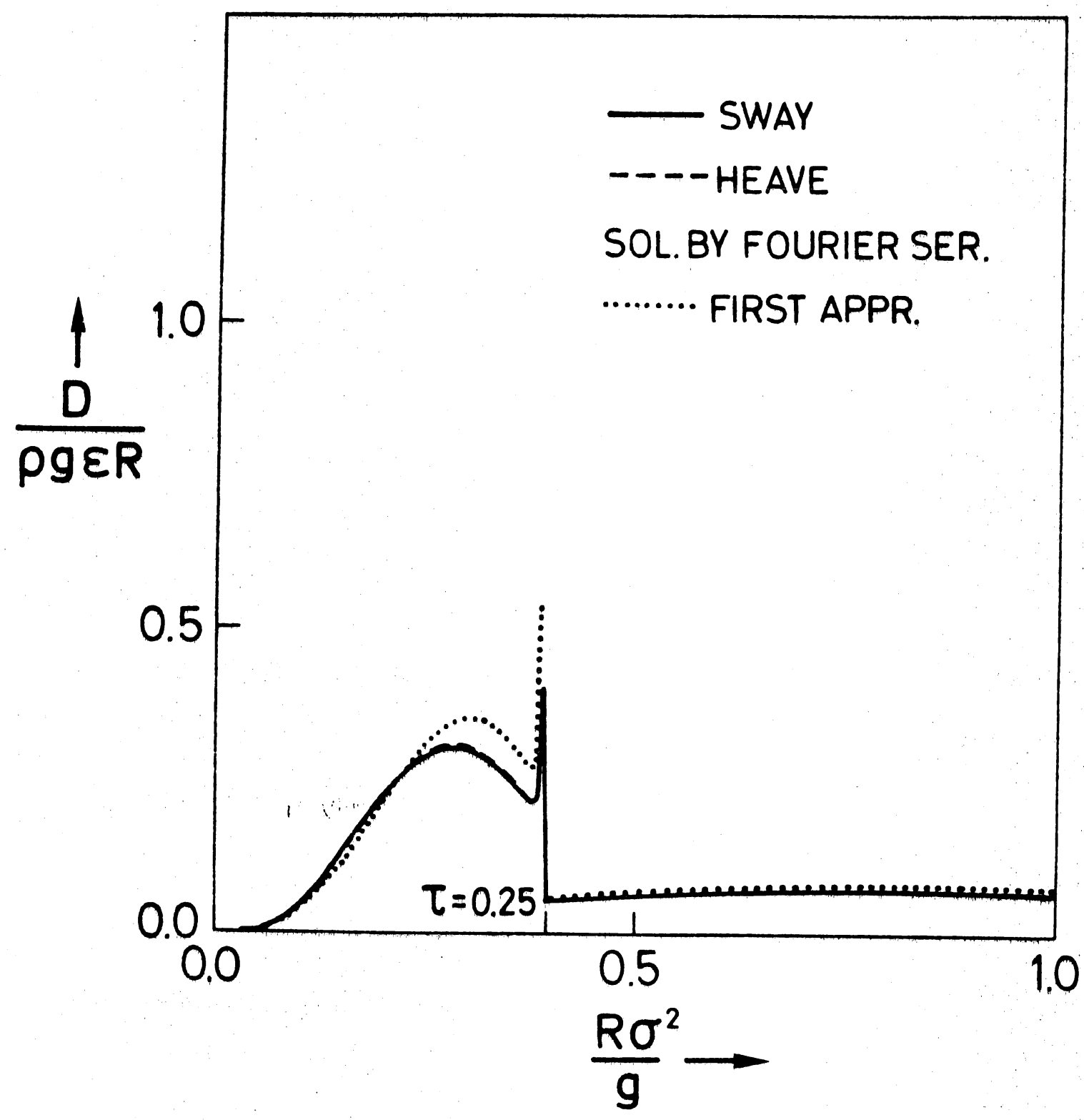

Figure 3c. Damping transfer functions in sway and heave, $d / R=3.0, U / \sqrt{q R}=0.4$. 



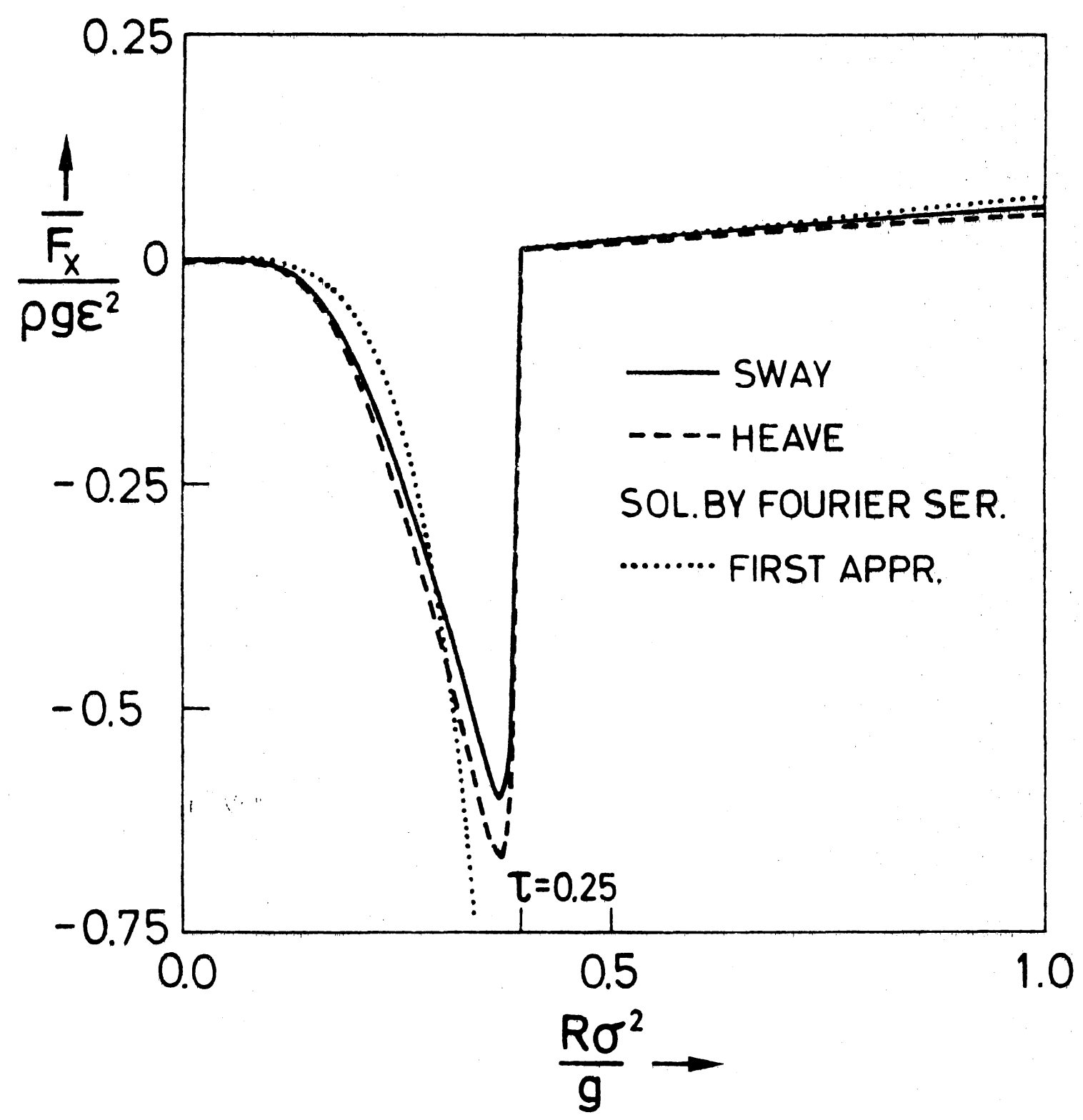

Figure 4. Mean second order horizontal force in sway and heave, $\mathrm{d} / \mathrm{R}=2.0, \mathrm{U} / \sqrt{\mathrm{gR}}=0.4$. 



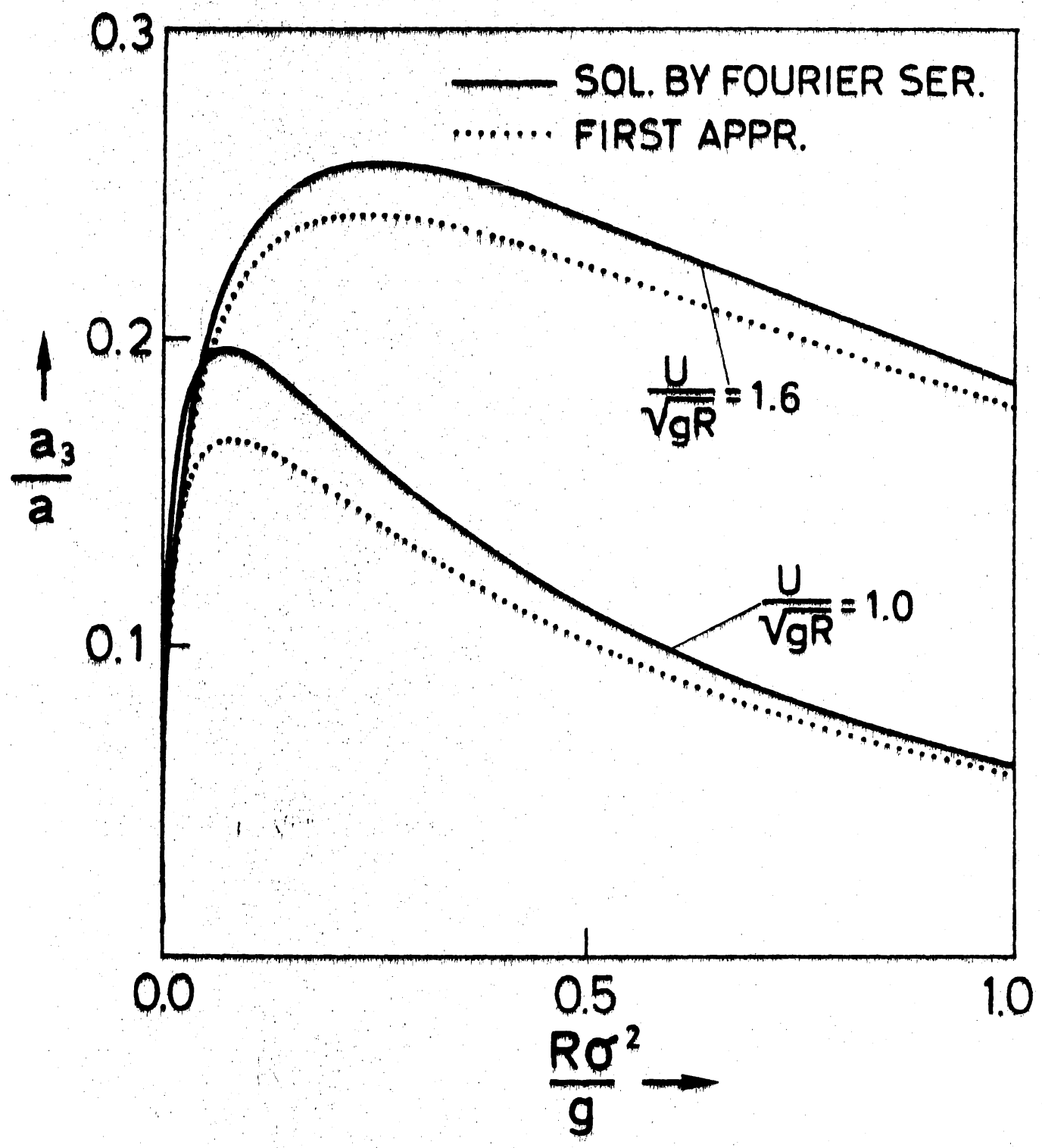

Figure 5a. Amplitude of new $\mathrm{k}_{3}$-wave in the diffraction problem when $k_{4}$-waves are incident upon the cylinder, $d / R=2.0, U / \sqrt{g R}=1.0,1.6$. 



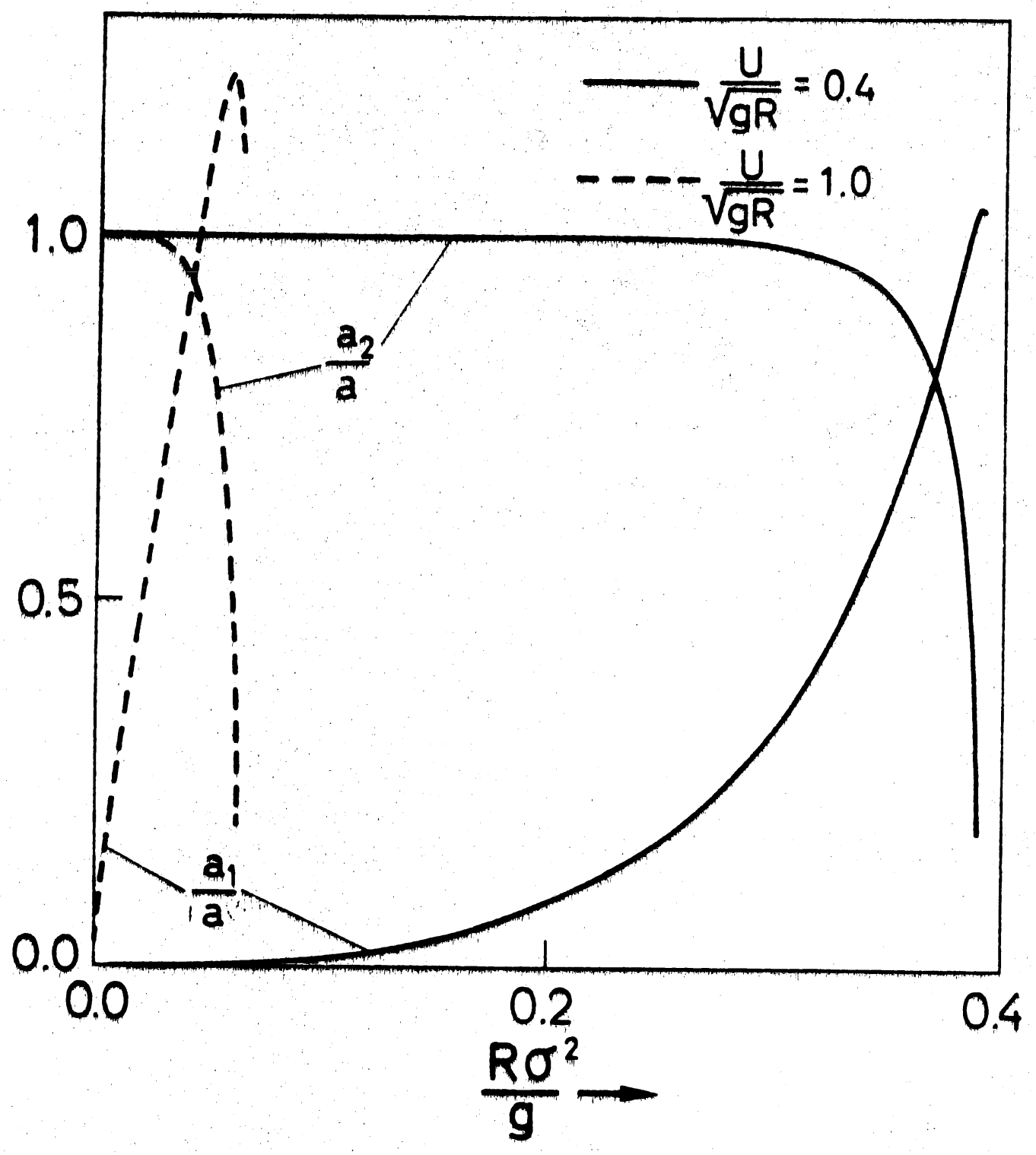

Figure 5b. Amplitude of transmitted $\mathrm{k}_{2}$-wave and reflected $k_{1}$ Twave when $k_{2}$-waves are incident upon the cylinder. $d / R=2,0, U / \sqrt{g R}=0.4,1.0$. 
$-$ 


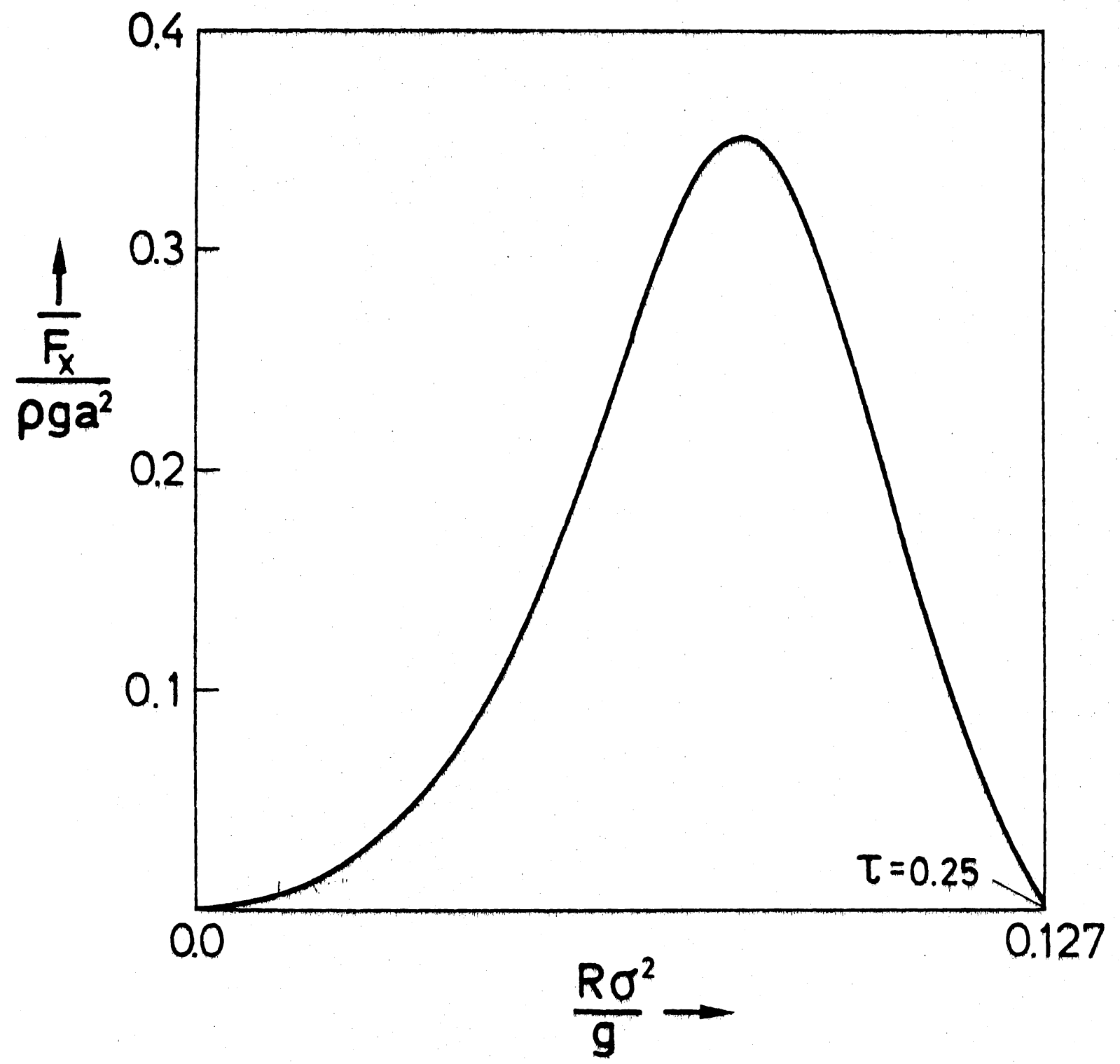

Figure 6 , Mean second order horizontal force when $\mathrm{k}_{2}$-waves are incident upon the cylinder, $d / R=2,0$, $\mathrm{U} / \sqrt{\mathrm{GR}}=0.7$. 


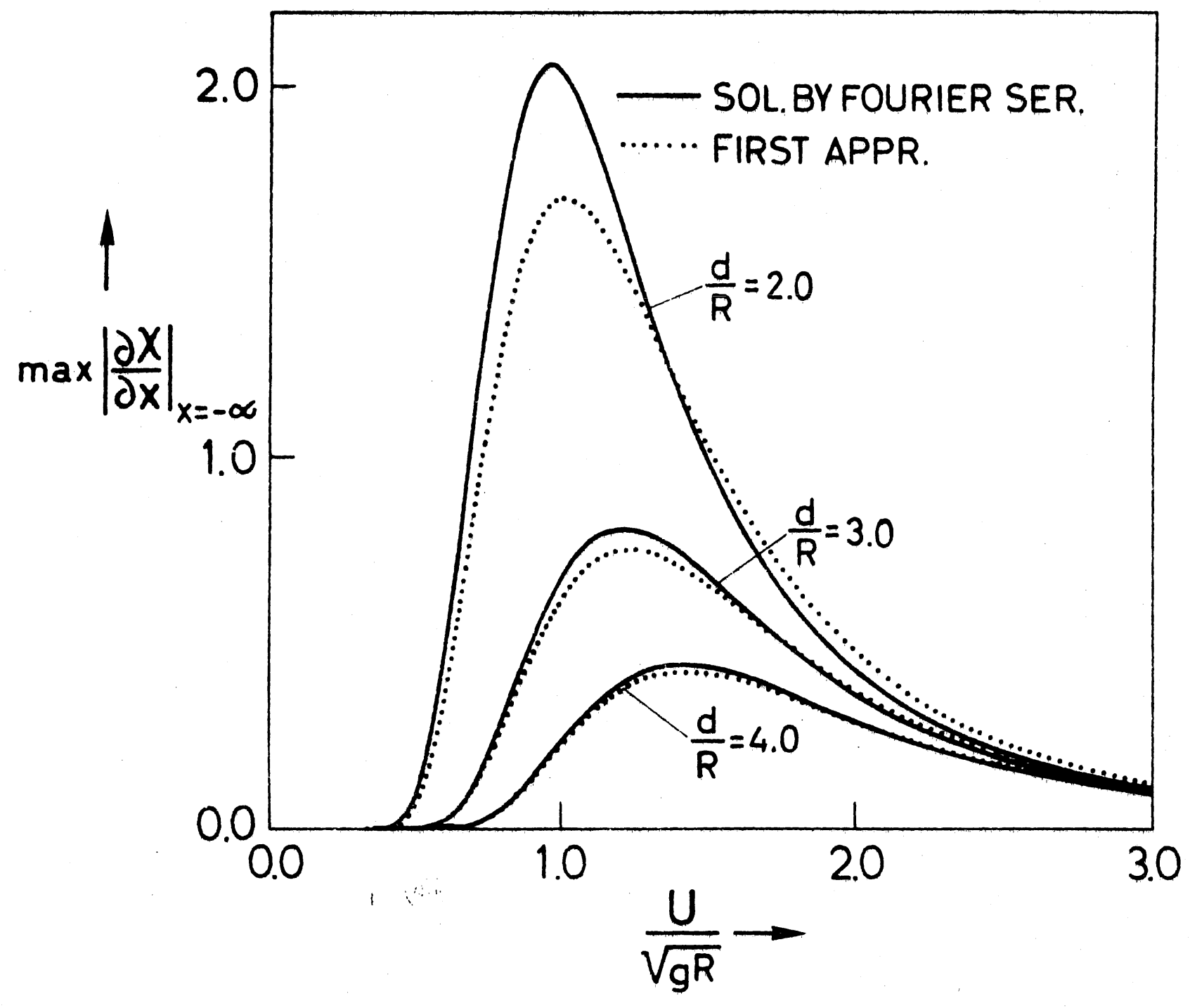

Fiqure 7. Maximum of $\mid \partial x / \partial x !$ at $x=-\infty$, for $d / R=2.0,3.0,4.0$. 
